\title{
A Meier-Gorlin Syndrome Mutation in Orc4 Causes Tissue-Specific DNA Replication Defects in Drosophila melanogaster
}

Stephen L. McDaniel, Anna M. Branstad, Allison J. Hollatz, Catherine A. Fox, and Melissa

M. Harrison

University of Wisconsin School of Medicine and Public Health, Department of Biomolecular Chemistry, Madison, WI 53706 
Running Title: MGS mutation causes replication defects.

Key Words: Drosophila, Meier-Gorlin syndrome, Disease Model, DNA Replication

Corresponding Author: Melissa M. Harrison, Department of Biomolecular Chemistry, 420

Henry Mall, 6204B Biochemical Sciences Building, Madison WI 53706. Email:

mharrison3@wisc.edu.Phone: (608) 262-2382. 


\section{Abstract:}

2 Meier-Gorlin syndrome is a rare recessive disorder characterized by a number of distinct

3 developmental defects, including primordial dwarfism, small ears, and small or missing

4 patella. Genes encoding members of the origin recognition complex (ORC) and additional

5 proteins essential for DNA replication (CDC6, CDT1, GMNN, CDC45, and MCM5) are

6 mutated in individuals diagnosed with MGS. The primary role of ORC is to license origins

7 during the $\mathrm{G} 1$ phase of the cell cycle, but it also plays roles in cilia development,

8 heterochromatin formation, and other cellular processes. Because of its essential role in

9 DNA replication, ORC is required for every cell division during development. Thus, it is unclear how the Meier-Gorlin syndrome mutations in ORC lead to the tissue-specific

11 defects associated with the disease. To address this question, we have used Cas9-

12 mediated genome engineering to generate a Drosophila melanogaster model of

13 individuals carrying a mutation in ORC4. Like the people with Meier-Gorlin syndrome,

14 these flies reach adulthood, but have several tissue-specific defects. Genetic analysis

15 revealed that this allele is a hypomorph and that mutant females are sterile. We demonstrated that this sterility is caused by a failure in DNA replication. By leveraging the

17 well-studied Drosophila system, we showed that a disease-causing mutation in orc4 18 disrupts DNA replication, and we propose that in individuals with MGS defects arise preferentially in tissues with a high-replication demand. 


\section{Introduction:}

Meier-Gorlin syndrome (MGS) is a rare developmental disorder. Individuals with MGS show developmental defects, including primordial dwarfism, small or missing patella, and small ears (Bicknell et al. 2011b; Guernsey et al. 2011; De Munnik et al. 2012). A significant number of patients also present with microcephaly, though typically have normal cognitive function (De Munnik et al. 2012). The first case of MGS was reported in 1959 (Meier et al. 1959) with a second case following in 1975 (Gorlin et al. 1975), but the underlying genetic cause of the disease was unknown. Recently, advancements in next-generation sequencing have enabled the identification of mutations causing MGS. The identified mutations are in a set of genes (ORC1, ORC4, ORC6, CDT1, CDC6, GMNN, CDC45, and MCM5) responsible for the function of DNA replication origins, the chromosomal positions required for the initiation of DNA replication (Bicknell et al. 2011b; Guernsey et al. 2011; De Munnik et al. 2012; Burrage et al. 2015; Fenwick et al. 2016; Vetro et al. 2017).

The average human undergoes $10^{16}$ cell divisions in a lifetime, and every cell division requires faithful duplication of the genome. Genome duplication begins at multiple individual DNA replication origins that are formed in a cell-cycle regulated multistep process requiring many proteins that are conserved throughout eukaryotic organisms (Remus and Diffley 2009). In G1-phase, origins are selected by the binding of the origin recognition complex (ORC) comprised of six highly conserved members (Orc1, Orc2, Orc3, Orc4, Orc5, and Orc6). ORC recruits Cdc6 and together this complex recruits the Cdt1 chaperone bound to the MCM hexamer, the replicative helicase. In an ATPdependent process, an MCM complex, comprised of two head-to-head hexamers 
44 (dhMCM), is formed on origin DNA, effectively 'licensing' the origin (Stillman 2005;

45 Sclafani and Holzen 2007; Remus et al. 2009). In S-phase, multiple proteins, including Sphase kinases and the MCM helicase accessory factors, Cdc45 and GINS, convert the

47 MCM complex into two active replicative helicases, which culminate in the initiation of DNA replication (origin function) (Moyer et al. 2006; Ilves et al. 2010). In most cell divisions, the genome must be replicated exactly once, and the cell-cycle separation of origin licensing (G1) and origin activation (S) ensures that only one complete round of genome duplication occurs per cell division (Deshaies 1995). However, in addition to this standard form of cell division, some cell-types undergo multiple rounds of genome duplication to generate polyploid cells (Lee et al. 2009). Both types of cell divisions depend on the same proteins for origin function.

As expected, based on the requirement for origin licensing for every cell division, null mutations in genes encoding proteins required for these processes are lethal (Micklem et al. 1993; Bell et al. 1993; Landis et al. 1997; Pinto et al. 1999; Pflumm and Botchan 2001; Shu et al. 2008; Park and Asano 2008; Baldinger and Gossen 2009; Balasov et al. 2009; Guernsey et al. 2011; Okano-Uchida et al. 2018). Thus, mutations in the genes that cause MGS must either be hypomorphic for their DNA replication functions or affect as yet undefined non-essential roles. Because origin function is essential in every cell division, it is unclear how MGS mutations that affect origin licensing result in tissuespecific defects. While ORC is essential for origin licensing, individual ORC subunits also function in other biological processes, such as heterochromatin formation (Prasanth et al. 2010) and cilia development (Hossain and Stillman 2012; Stiff et al. 2013). Thus, it is 
To determine the molecular mechanism underlying MGS mutations, MGS models have been generated in several organisms. In particular, the Orc4 MGS mutation has been generated in Saccharomyces cerevisiae (Sanchez et al. 2017). Yeast with this Orc4 (Y232C) substitution grow slowly due to defects in replication of the ribosomal DNA (rDNA) locus that normally contains hundreds of copies of the $9 \mathrm{~kb}$ rDNA locus, each with

72 its own origin. In orc $4^{Y 232 C}$ yeast cells, the rDNA origin is insufficiently functional leading to DNA replication stress that causes substantial decreases in rDNA copy number, and the reduced translational capacity is not able to support a normal growth rate. Thus, while the orc $4^{\mathrm{Y} 232 \mathrm{C}}$ allele clearly causes DNA replication defects at some yeast origins, it is challenging to determine whether the slow-growth phenotype is due to replication defects per se or the downstream effects on translation. In Drosophila, a transgenic system has evidence that these phenotypes are due to a destabilization of ORC, which in turn results in decreased recruitment of the MCM hexamer to chromatin (Bleichert et al. 2013).

82 Replication defects are also evident in cultured cells derived from MGS patients with multiple different ORC1 alleles (Hossain and Stillman 2012). In contrast to the replication defects identified in yeast and flies, cell culture and zebrafish models for Orc1 mutants show defects in cilia development and these defects in turn may generate the various morphological phenotypes observed in transgenic fish models. (Bicknell et al. 2011b; Hossain and Stillman 2012; Yao et al. 2017; Maerz et al. 2019). Thus, while these models have provided important insights into this pleotropic disease, it remains unclear whether the different mechanisms identified reflect differences in the underlying causes of MGS 
90 or whether they reflect differences in how the mutations were modelled. Finally, it is also

91 important to note that the metazoan models to date rely on exogenous expression of the

92 mutant protein and therefore do not precisely mimic the conditions observed in MGS

93 individuals.

To better understand the molecular mechanisms underlying the tissue-specific

95 phenotypes caused by MGS mutations, we used Cas9-genome editing to generate a

96 Drosophila model of MGS. Because there is a single identified MGS mutation in a highly

97 conserved region of Orc4 (Y174C), we generated a Drosophila model for this mutation

98 and demonstrated that similar to the disease phenotype, flies homozygous for this

99 mutation are viable. In addition, we created a wild-type control and a null mutant allele,

100 which enabled us to demonstrate that the MGS mutation is a hypomorphic allele that

101 causes tissue-specific replication defects resulting in female sterility. Together our data

102 suggest that the tissue-specific defects identified in MGS patients may result from cells

103 within these tissues having a high-replication demand that cannot be met by the MGS

104 mutant replication factors. Further, this work demonstrates the power of genome-editing

105 in the fly to model human disease. 


\section{Materials and Methods:}

Fly lines and husbandry: Flies were grown on standard molasses food at $25^{\circ} \mathrm{C}$. Fly lines used in this study: orc4 ${ }^{W T}$ (this study), orc4 ${ }^{Y 162 C}$ (this study), orc4 null (this study), $m c m 6^{K 1214} v[24] / F M 3$ (Bloomington Drosophila Stock Center (BDSC) \#4322),

Generation of orc4 ${ }^{W T}$ and orc4 $4^{Y 162 C}$ fly lines: Single-stranded donor oligonucleotides (ssODNs) were generated to target the region of orc4 encoding Y162. Each ssODN had silent mutations to mutate the PAM site and generate a novel Ndel cut site for molecular screening. The orc $4^{\mathrm{Y} 162 \mathrm{C}}$ ssODN also contained the necessary alterations to create the Y162C mutation. The orc $4^{W T}$ ssODN did not contain this mutation. gRNA plasmids and ssODNs were mixed, injected, and screened as in (Hamm et al. 2017). Injections were done by Best Gene Inc.

orc ${ }^{W T}$ SSODN:

122 AAGAGAAAGACCTGCCGGTGCGAGAAACGCGACTTGACCCGCTTCTCCAGCAGCT 123 CGATCACGTCGAGGCGACAGGTAACGCCAAGTACACATATGGGCGCCTGGGCTA CTGGGAGACGTCGAAGAGGTTGTAAAGCAGGGTCTGGTTGTGGTGAGCACAGAA GAGGTCGAACTCCTCGAGAAT

orc4 $^{\text {Y162C }}$ SSODN:

AAGAGAAAGACCTGCCGGTGCGAGAAACGCGACTTGACCCGCTTCTCCAGCAGCT CGATCACGTCGAGGCGACAGGTAACGCCAAGTACACATATGGGCGCCTGGGCTA CTGGGAGACGTCGAAGAGGTTGCAAAGCAGGGTCTGGTTGTGGTGAGCACAGAA GAGGTCGAACTCCTCGAGAAT 
Viability Assays: 3-5 heterozygous males and 5-10 heterozygous females of the indicated genotypes were mated in standard molasses vials with dry yeast and flipped twice at 2day intervals. Two days after the final flip, the adult flies were cleared from the vials and their progeny were allowed to reach adulthood. Over 800 adults were counted for each cross. The ratio of $\mathrm{CyO}$ and non-CyO adults was determined and the $X^{2}$ value was

141 calculated for each cross, correcting for the observed ratio from the orc4WT/CyO self142 cross.

Adult Phenotyping: Adult flies from the indicated genotypes were imaged on a Nikon SMZ745 dissection microscope (back bristles) or frozen at $-20^{\circ} \mathrm{C}$ and then imaged on a Zeiss Axioplan2 epifluorescence microscope (wing bristles).

Ovary DAPI Staining: Females of the indicated genotypes were mixed with males in molasses vials with a small amount of yeast paste and grown for two days. Flies were flipped into a fresh vial after 24 hours. Ovaries were dissected into Grace's medium. The

151 media was removed, and the ovaries were resuspended in $0.5 \mathrm{ml}$ of fix solution (4\% 152 formaldehyde in 1xPBS) and incubated for 15 minutes at room temperature while rocking.

153 The ovaries were washed twice with $1 \mathrm{ml}$ of PBST (1XPBS $+0.2 \%$ Triton X-100) and then 154 washed for 5 minutes with 1 XPBS to remove the detergent. Ovaries were then incubated 155 with 1xPBS + DAPI (1:1000) for 15 minutes and then mounted on a slide, covered with a 156 coverslip, and sealed with clear nail polish. Ovaries were imaged on Zeiss Axioplan2 157 epifluorescence microscope. 
159 Nurse Cell Counts: DAPI stained ovaries from the indicated genotypes were imaged on a Zeiss Axioplan2 epifluorescence microscope, and the nurse cells were counted. Nurse cells from 50-100 stage 10 egg chambers were counted for each genotype.

EdU Assay: Ovaries from ten females were dissected as described above and resuspended in 100 ul of Grace's media. 100 ul of 2XEdU in Grace's media (15 uM) stock solution was added to each sample and incubated for 1.25 hours at room temperature. The ovaries were washed twice with 200 ul of $3 \%$ BSA in 1XPBS for 5 minutes each time.

167 The ovaries were then fixed for 15 minutes in 200 ul of $4 \%$ formaldehyde in 1 XPBS. After fixation, the ovaries were washed twice with 200 ul of PBST (1XPBS $+0.5 \%$ Triton X100) for 5 minutes and 20 minutes. Ovaries were then washed twice for 5 minutes each with $1 \mathrm{XPBS}+3 \% \mathrm{BSA}$ and then carried through the Click-iT Plus EdU Imaging Kit

171 protocol (Thermo Fisher Scientific). Ovaries were imaged on a Nikon A1R-SI+ confocal 172 microscope and >50 stage 10 chambers were examined for EdU foci in each genotype.

174 Generation of Germline Mitotic Clones and Egg Counting: FRTG13 orc4 ${ }^{\text {Y162C }} / \mathrm{CyO}$ 175 females were mated with $h s F L P 122 ; P\{w[+m W . h s]=F R T(w[h s])\} G 13 P\{w[+m C]=o v o D 1-$ $17618\} 2 R / T(1 ; 2) O R 64 / \mathrm{CyO}$ males. Their progeny were incubated for 30 minutes at $37^{\circ} \mathrm{C}$ in 177 a circulating water bath either 24-48 hours after egg laying (1x heat shock) or 24-48 and 178 48-72 hours after egg laying (2x heat shock). These embryos were reared to adulthood. 179 Non-CyO females were isolated and mated to males in standard molasses vials. 180 Homozygous orc $4^{W T}$ and orc $^{\mathrm{Y162C}}$ females were also mated to males as controls. The 
181 crosses were flipped every 24 hours for four days, and the number of eggs laid each day

182 were counted.

183

184 Data availability

185 Strains and plasmids are available upon request. The authors affirm that all data

186 necessary for confirming the conclusions of the article are present within the article,

187 figures and tables. 


\section{Results:}

\section{Generating endogenous point mutations in Drosophila to model Meier-Gorlin}

\section{syndrome}

Meier-Gorlin syndrome (MGS) results from mutations in multiple different origin regulatory proteins, each of which is required for replication during every cell division of the organism (Bicknell et al. 2011b; Guernsey et al. 2011; De Munnik et al. 2012). Given the additional cellular roles of these factors, we sought to model the disease in a rapidly developing metazoan to better understand the mechanisms behind the phenotypic defects. The only mutation in ORC4 that causes MGS is the substitution of tyrosine 174 to cysteine (Guernsey et al. 2011). Tyrosine 174 is in the AAA+ ATPase domain of ORC4 (Figure 1A). This region is highly conserved from yeast to humans, suggesting that it plays a critical role in ORC function and enabled us to identify the homologous residue (tyrosine 162) in Drosophila.

We used Cas9-mediated genome engineering to introduce a mutation encoding a tyrosine to cysteine substitution (Y162C) at the endogenous orc4 locus. Mutating the endogenous locus assured that any phenotypic changes observed were due to the mutation and not to changes in transcription that might be caused by transgenic constructs. In addition to the $\mathrm{Y} 162 \mathrm{C}$ substitution, our genome engineering strategy introduced a silent mutation in the protospacer adjacent motif (PAM) to inhibit the guide RNA from directing cleavage of the engineered genome. We also engineered a silent mutation introducing a Ndel restriction site to allow for rapid molecular screening of the modified genomes (Figure 1B). To ensure that the two silent mutations do not affect Orc4 activity, we also generated a control strain, orc4 ${ }^{W T}$, which does not have the $\mathrm{Y} 162 \mathrm{C}$ 
211 substitution, but includes both silent mutations. In addition, our editing generated a likely

212 null allele, orc $4^{\text {null }}$, through a single base pair deletion resulting in a frameshift after I187.

213 While we have not analyzed protein product from this allele, we presume that this is a null

214 allele based on the molecular nature of the mutation and the fact that, similar to null alleles

215 in other ORC subunits, homozygous orc4 ${ }^{\text {null }}$ animals are not viable (Landis et al. 1997;

216 Pinto et al. 1999; Pflumm and Botchan 2001; Park and Asano 2008; Baldinger and

217 Gossen 2009; Balasov et al. 2009). Together, these strains provide the first metazoan

218 model for an MGS mutation in which the mutation was engineered at the endogenous

219 locus along with precisely defined control strains.

220

221

\section{orc $^{\mathrm{Y} 162 \mathrm{C}}$ is a hypomorph and animals are homozygous viable}

222

MGS individuals with ORC4 $4^{Y 174 C}$ mutations are either homozygous for this

mutation or carry it over a null allele (Bicknell et al. 2011a; Guernsey et al. 2011; De

Munnik et al. 2012). While these individuals possess a number of distinctive phenotypes,

they survive and have a normal expected lifespan. Therefore, we initially tested the

viability of homozygous orc $4^{\mathrm{Y162C}}$ animals. We quantitatively assessed the number of non- 
2001; Park and Asano 2008; Baldinger and Gossen 2009; Balasov et al. 2009) (Figure 1C). Like individuals carrying the $\mathrm{ORC} 4^{\mathrm{Y} 174 \mathrm{C}}$ mutation, orc4 $4^{\mathrm{Y162C}}$ animals are homozygous viable. Unlike some prior models of MGS mutations (Bicknell et al. 2011b; Yao et al. 2017; Maerz et al. 2019), we did not observe any reduction in size of the homozygous orc4 ${ }^{\text {Y162C }}$ animals, suggesting either a difference between Drosophila and other organisms or that some of these phenotypes may have been caused by misexpression of the disease allele which was avoided by our genome-editing strategy. When we corrected our expected ratio of $C y O$ to non-CyO flies based on the observed ratio from the orc $4^{W T} / C y O$ cross, we identified a statistically significant decrease in the number of orc $4^{\mathrm{Y162C}} / \mathrm{orc}^{\mathrm{Y} 162 \mathrm{C}}$ adults $\left(x^{2}, p=5.0 \times 10^{-6}\right)$.

The observed impact on viability of the homozygous orc $4^{\mathrm{Y} 162 \mathrm{C}}$ mutation suggested it might be a loss-of-function allele. To directly test this, we scored the viability of orc $4^{\mathrm{Y} 162 \mathrm{C} /}$ orc $4^{\text {null }}$ trans-heterozygotes. These flies reach adulthood at reduced levels as compared to orc $4^{Y 162 C}$; we recovered one third of the expected number of trans-heterozygotes $\left(\mathrm{X}^{2}\right.$, $p=1.1 \times 10^{-60}$ ), demonstrating that orc $4^{\mathrm{Y} 162 C}$ is a hypomorphic allele. Together, these data showed that similar to MGS patients, the homozygous orc $4^{\mathrm{Y} 162 \mathrm{C}}$ animals are viable. Furthermore, our genetic analysis demonstrated that the tyrosine to cysteine substitution in Orc4 likely results in a protein with reduced functionality.

\section{Animals homozygous for orc $4^{\mathrm{Y} 162 \mathrm{C}}$ have tissue-specific defects}

Given that ORC is required during replication of every cell, an outstanding question regarding the phenotypes of individuals with MGS is why there are tissue-specific defects. Having demonstrated that orc4 ${ }^{\mathrm{Y} 162 \mathrm{C}}$ animals reach adulthood, we were able to assay for 
257 tissue-specific phenotypes. When compared to a wild-type strain $\left(w^{1118}\right)$, orc $4^{W T}$ animals 258 show no obvious phenotypic differences. Thus, we used these as our wild-type controls 259 throughout the remainder of the study. By comparison, orc $4^{\mathrm{Y} 162 \mathrm{C}}$ homozygous animals 260 had several phenotypic abnormalities. We identified several missing bristles on the thorax 261 (Figure 2A). In addition, we observed severe bristle defects on the wing. The bristles 262 along the wing are normally uniform in length and evenly spaced. By contrast, the wing margin bristles in the orc $4^{\mathrm{Y} 162 \mathrm{C}}$ animals are disorganized and vary in length along the wing

264 (Figure 2A). Therefore, orc $4^{\mathrm{Y} 162 \mathrm{C}}$ homozygous animals resemble individuals with MGS as 265 they possess tissue-specific defects, further strengthening the relevance of our 266 Drosophila model.

\section{Females homozygous for orc $4^{\mathrm{Y} 162 \mathrm{C}}$ are sterile}

While orc4 ${ }^{\mathrm{Y} 162 \mathrm{C}}$ animals were homozygous viable, females were sterile. By contrast, homozygous orc $4^{\mathrm{Y} 162 \mathrm{C}}$ males were fertile. Females produced eggs at very low

271 frequencies compared to orc $4^{W T}$ control animals. The few eggs produced did not have 272 dorsal appendages and appeared watery and malformed, indicative of a thin eggshell.

273 Indeed, female sterility is a phenotype shared amongst animals possessing loss-of-

274 function mutations in a variety of replication factors, such as Orc2, MCM6, Cdt1, and 275 chiffon (Orr-Weaver 1991; Landis et al. 1997; Landis and Tower 1999; Whittaker et al. 276 2000).

277 Because the females are sterile, we examined the ovaries of orc4 ${ }^{\text {Y162C }}$ 278 homozygous animals to determine if there were specific defects in egg chamber 279 development. We dissected ovaries from orc4 ${ }^{W T}$ and $\operatorname{orc}^{\mathrm{Y} 162 C}$ homozygous females and 
stained them with 4', 6-diamidino-2-phenylindole (DAPI) to image the nuclei. The ovarioles appeared largely normal, and we could identify egg chambers through stage 14. Nonetheless, we noted two distinct phenotypes in stage 10 chambers: disrupted follicle-cell patterning and decreased numbers of nurse cells (Figure 2B). Both cell types play critical roles in oocyte maturation, and the deleterious phenotypes we observed could be responsible for the female sterility. The somatically derived follicle cells rapidly amplify the chorion genes during stage 10B (Orr-Weaver 1991). Simultaneously, the nurse cells produce the maternally derived products that will be deposited into the egg. Both cell types are polyploid, and the disorganized follicle cell structure and decreased nurse cell numbers could potentially be due to replication defects during oocyte maturation.

To more quantitatively assess these defects, we determined the number of nurse cells in orc $4^{W T}$, orc $4^{Y 162 C}$, and $m c m 6^{K 1214}$ homozygous females. We included $m c m 6^{K 1214}$ as a control since it is a female-sterile allele of an additional component of the replication machinery (Komitopoulou et al. 1983). Using fixed, DAPI stained ovaries, we counted the number of nurse cells in 50-100 stage 10 egg chambers. Wild-type stage 10 egg chambers possess 15 nurse cells. Ovaries from orc $4^{Y 162 C}$ homozygous females have fewer nurse cells than orc $4^{W T}$ females along with a wider distribution in the number of nurse cell per egg chamber (t-test, $p=4.16 \times 10^{-12}$ ) (Figure 2C). By contrast, stage 10 chambers from the mcm6 $6^{K 1214}$ females showed only a minor decrease in nurse cell number as compared to ovaries from orc $4^{W T}$ females (t-test, $p=7.79 \times 10^{-3}$ ) and were significantly different in comparison to $\operatorname{orc}^{Y 162 C}\left(t\right.$-test, $\left.p=1.03 \times 10^{-10}\right)$. Thus, while both the orc $4^{\mathrm{Y162C}}$ and $m c m 6^{K 1214}$ alleles lead to female sterility, only orc $4^{\mathrm{Y162C}}$ females have 
a decreased number of nurse cells. This result provides evidence that the sterility of orc ${ }^{\mathrm{Y} 162 \mathrm{C}}$ females may result from a defect in cells distinct from the nurse cells.

\section{Females homozygous for orc $4^{\mathrm{Y} 162 \mathrm{C}}$ fail to amplify the chorion genes}

Mutations in genes encoding replication factors are known to cause female sterility at least in part due to replication defects in the somatic follicle cells. At stage 10B, follicle cells in the egg chamber undergo endoreplication, selectively amplifying a limited subset of loci including the chorion genes, which are essential for eggshell production later during oocyte maturation (Orr-Weaver 1991; Calvi et al. 1998). This results in a gene amplification of $16-20$ fold for a region on the $\mathrm{X}$ chromosome and $60-80$ fold for a region of the $3^{\text {rd }}$ chromosome. Amplification occurs through repeated rapid and precise rounds of origin firing and replication fork elongation. Failure to adequately amplify these loci leads to thin, fragile eggshells, which results in female sterility. Thus, the female sterility of orc $4^{\mathrm{Y} 162 \mathrm{C}}$ may result from a failure to adequately amplify the chorion genes during oocyte maturation. Furthermore, well-established assays for chorion gene amplification provide a system by which to directly assay whether the MGS mutation in orc4 impacts DNA replication (Park and Asano 2012).

To test if orc $4^{\mathrm{Y} 162 \mathrm{C}}$ animals are replication deficient, we dissected ovaries from $\operatorname{orc}^{W T}$, orc $4^{\mathrm{Y} 162 \mathrm{C}}$, and $m c m 6^{K 1214}$ homozygous females and incubated them with the modified thymidine analogue EdU for 1.25 hours, which allowed for the incorporation of EdU into replicating DNA that could subsequently be imaged using a small moleculebased fluorescent assay. Amplification of the chorion gene loci can be visualized as distinct EdU foci in the follicle cells of stage 10B egg chambers. Indeed, in the orc $4^{W T}$ 
325 females we observed large robust foci in the follicle cells (Figure 3A). As expected, no foci were detected in stage 10 egg chambers from mcm6 $6^{K 1214}$ ovaries (Figure 3A). Despite clear incorporation of EdU in other stages of egg chamber development, no EdU foci were evident in the stage 10 egg chambers of orc $4^{\mathrm{Y} 162 \mathrm{C}}$ females (Figure $3 \mathrm{~A}$ ). To quantify this replication defect, we imaged $>50$ stage 10 egg chambers for each genotype. There were large robust foci in $45 \%$ of the orc $4^{W T}$ stage 10 egg chambers, but none in orc $4^{Y 162 C}$ animals ( $t$-test, $p=2.67 \times 10^{-11}$ vs. orc $4^{W T}$ ) (Figure $3 B$ ). In ovaries from $m c m 6^{K 1214}$ females, we identified weak, fractured foci in $2 \%$ of the stage 10 egg chambers $\left(t\right.$-test, $p=4.60 \times 10^{-8}$ vs. orc $4^{W T}$ ). We noted that the Hoechst signal for the orc $4^{\mathrm{Y} 162 \mathrm{C}}$ animals was lower as compared to either the orc $4^{W T}$ or $m c m 6^{K 1214}$ animals. While this could be an issue with

335 the staining, all the samples were stained at the same time with the same reagent mix.

336 Thus, it is possible that this reflects a lower DNA content in the follicle cells of the orc $4^{Y 162 C}$

337 females as compared to control animals. Because the follicle cells themselves undergo a 338 few rounds of total endoreplication cycles, it is possible this difference in Hoechst staining reflects a decrease in this endoreplication. Together these data provide evidence that the $\operatorname{orc}^{\mathrm{Y162C}}$ mutation leads to a specific replication defect as females fail to amplify the chorion gene locus and this in turn results in the observed thin, fragile eggshells. Thus,

342 while orc $4^{\mathrm{Y} 162 \mathrm{C}}$ mutant animals can replicate their DNA, DNA replication is defective 343 during chorion gene amplification where there may be a high demand for rapid origin 344 licensing and firing.

\section{Animals inheriting maternal orc $4^{\mathrm{Y} 162 \mathrm{C}}$ cannot complete embryogenesis}


rapid origin licensing and firing is during the synchronous nuclear divisions in the early embryo. Immediately following fertilization, development is controlled by maternally deposited products while the genome is reprogrammed. During this time, the nuclei are in a shared, syncytial cytoplasm and divide quickly with an abbreviated cycle comprised of only a synthesis (S) phase and mitosis. These divisions occur approximately every 10 minutes with DNA being replicated in about half of this time. We hypothesized that if the tyrosine-to-cysteine mutation in Orc4 caused defects in tissues in which there was a high demand for DNA replication that embryos inheriting maternal orc $4^{\mathrm{Y} 162 \mathrm{C}}$ would fail to progress through the early stages of development. To address this, we used the FLP/FRT system to generate germline clones that are homozygous for the orc ${ }^{\mathrm{Y} 162 \mathrm{C}}$ mutation, while remaining largely heterozygous for the mutation in the somatic follicle cells (Chou and Perrimon 1996). Because we combined this with the dominant female-sterile ovo ${ }^{D 1}$ mutation (Chou et al. 1993), this strategy generated animals that only inherited orc4 ${ }^{\mathrm{Y} 162 \mathrm{C}}$ maternally. Females heterozygous for both the ovo ${ }^{D 1}$ and the $o r c 4^{\mathrm{Y} 162 \mathrm{C}}$ mutations did not produce eggs, as expected. By contrast, heat-shocked females laid some eggs, and these eggs had dorsal appendages, a striking difference from the very few eggs laid by orc4 ${ }^{\mathrm{Y} 162 \mathrm{C}}$ homozygous females (Figure $4 \mathrm{~A}$ ). This suggests that our strategy at least partially rescued eggshell production. We quantified the numbers of eggs laid by females in which the germline clones (glc) were generated. These females laid $\sim 4$ times more eggs than orc $4^{\mathrm{Y} 162 \mathrm{C}}$ females (Figure 4B). While these embryos were rescued for eggshell production, they failed to complete embryogenesis and showed general morphological defects (Figure 4A). This failure to progress through embryogenesis further supports the model that the hypomorphic orc $4^{\mathrm{Y} 162 \mathrm{C}}$ mutation results in replication defects in tissues that 
bioRxiv preprint doi: https://doi.org/10.1101/711820; this version posted July 23, 2019. The copyright holder for this preprint (which was not certified by peer review) is the author/funder, who has granted bioRxiv a license to display the preprint in perpetuity. It is made available under aCC-BY-NC-ND 4.0 International license.

371 require rapid origin licensing and firing and that it is this tissue-specific requirement that

372 leads to the distinct phenotypes of MGS individuals. 


\section{Discussion:}

The underlying mechanisms generating the phenotypes of MGS individuals are

not well understood. While the mutations that lead to this disease are clustered in genes required for the licensing of origins of replication during the G1 phase of the cell cycle, ORC has additional functions outside of DNA replication. Using Cas9-mediated mutagenesis, we have engineered the endogenous orc4 locus to establish a metazoan model for MGS. Our Drosophila model recapitulates the disease state as animals are homozygous viable, demonstrating that the encoded tyrosine-to-cysteine mutation in Orc4 is compatible with DNA replication in many tissues. Genetic analysis showed that orc $4^{\mathrm{Y} 162 \mathrm{C}}$ is a hypomorph that is predicted to generate a protein with reduced functionality. Indeed, orc $4^{Y 162 C}$ females are sterile, and this is likely due to a replication defect in tissues with a high-replication demand, like the ovarian follicle cells. Together our data provide a mechanistic model for MGS phenotypes and, in so doing, demonstrate the utility of the well-established Drosophila system for rapidly generating metazoan disease models and testing specific mechanistic hypotheses to explain the observed patient phenotypes.

In this study, we demonstrate that the orc $4^{\mathrm{Y162C}}$ mutation results in defects in two distinct tissues with high-replication demand (the early embryo and chorion gene amplification in the follicle cells). Based on these data, we propose that the MGS mutation in Orc4 generates a sub-functional ORC that cannot meet the replication demands, resulting in defects specifically in tissues requiring fast/efficient replication, which are particularly sensitive to compromised replication machinery. While we propose that replication speed is a critical factor that leads to the phenotypic defects in orc4 ${ }^{\mathrm{Y} 162 \mathrm{C}}$ animals, we cannot exclude the possibility that the defects are due to the non-canonical 
cell cycles in the tissues assayed. In the nurse and follicle cells of the ovary and in the early embryo, the replication cycle does not occur with the standard, four phases (G1, S, G2, M). Instead, the nurse cells undergo multiple rounds of complete genome endoreplication. In the follicle cells, the chorion gene loci undergo multiple rapid rounds of re-initiation of DNA synthesis. Similarly, in the early embryo the nuclear division cycle is a series of rapid synthesis and mitosis phases without gap phases. Further experiments will be required to determine if phenotypes caused by MGS mutations are due specifically to defects in tissues with cycles of rapid DNA replication, non-standard cycles of DNA replication, or both.

What is clear is that orc $4^{\mathrm{Y} 162 \mathrm{C}}$ flies are defective in replicating the chorion gene loci and that this causes female sterility. Similarly, yeast with the corresponding tyrosine-tocysteine mutation (Y232C) show replication defects, particularly at the ribosomal DNA locus origin (Guernsey et al. 2011; Sanchez et al. 2017). Only this tyrosine-to-cysteine mutation at position 174 has been associated with MGS, suggesting that a very limited subset of mutations can be tolerated for viability and that this may be the one of few permissible mutations at this locus. From recent structural studies, it is evident that the region of Orc4 surrounding and including Y174 interacts with the ATPase domain of Orc1 with Y174 of Orc4 making contacts with E621 of Orc1 (Tocilj et al. 2017). This domain of Orc4 is highly conserved across species, suggesting there is significant evolutionary constraint placed on these residues. This likely reflects a functional requirement for the interaction between Orc4 and Orc1 in this region. Indeed, the tyrosine-to-cysteine mutation in human Orc4 results in altered ORC ATPase activity in vitro (Tocilj et al. 2017). Because ORC ATPase activity is essential for origin function, these data, combined with 
419 our in vivo observations, provide a compelling molecular model in which the orc4 $4^{\text {Y162C }}$ 420 mutation leads to a functional, but compromised ORC that results in decreased

421 recruitment of the MCM hexamer to chromatin. Because most cells license more origins

422 of replication than are necessary to replicate the genome, this reduction in MCM loading

423 can be tolerated in most tissues. However, in tissues that require rapid or efficient rounds

424 of replication, the decrease in MCM loading may result in replication defects. Indeed, 425 limiting amounts of the MCM helicase can have tissue-specific defects as demonstrated 426 by the fact that mice with a hypomorphic allele of MCM3 die from anemia due to 427 replication failure of the rapidly dividing erythrocyte precursor cells (Alvarez et al. 2015). Together these data from multiple model systems suggest that in individuals with 429 the Orc4 Y174C MGS mutation have an insufficient number of origins to support rapidly 430 replicating tissues. Nonetheless, it remains unclear if MGS mutations in other ORC 431 subunits similarly result in decreased replication capacity in specific tissues. Biochemical 432 and phenotypic data from Drosophila suggest that the MGS mutation in orc6 results in a 433 replication defect caused by a destabilization of ORC (Bleichert et al. 2013; Balasov et al. 434 2015). Similar to the model we propose for Orc4 Y174C, this destabilized ORC decreases 435 the recruitment of the MCM hexamer to chromatin (Bleichert et al. 2013). Modelling of 436 the Orc1 MGS mutations in zebrafish generates small fish with morphological defects, 437 similar to the phenotypes in MGS individuals. In contrast to the models proposed for Orc4 438 and Orc6, the phenotypes in the zebrafish model of Orc1 MGS mutations have been 439 suggested to arise from defects in cilia formation (Bicknell et al. 2011b; Yao et al. 2017; 440 Maerz et al. 2019). Thus, it remains possible that roles for ORC proteins outside of origin 441 licensing may impact MGS phenotypes. Future work in multiple organisms will be needed 
442 to identify how each MGS mutation leads to the disease phenotypes. Nonetheless, our

443 data clearly demonstrate tissue-specific replication defects caused by the MGS-

444 associated mutation in Orc4. Thus, the tissue-specific defects in MGS patients may arise

445 because cells within these tissues have specialized replication demands. Furthermore,

446 our data demonstrate the ability to gain mechanistic insights into disease phenotypes by

447 combining rapid and precise editing of the Drosophila genome with the wealth of tools

448 and knowledge derived from over a century of studying this powerful model metazoan. 
Figure Legends:

451 Figure 1: Similar to MGS individuals, orc $4^{\text {Y162C }}$ animals are viable (A) Schematic of

452 Drosophila Orc4 protein domains with the AAA+ ATPase domain (AAA) and the winged 453 helix (WH) domain indicated. The Walker A (WA), Walker B (WB), Sensor 1 (S1) and 454 Sensor 2 (S2) motifs are also indicated in the AAA+ ATPase domain (top). Clustal Omega 455 alignment of multiple eukaryotes for the region surrounding the conserved tyrosine 456 residue mutated in MGS (highlighted). (bottom) (B) Sequence of the genomic region 457 encoding Y162 of Orc4 from a wild-type, unedited Drosophila strain (top). Below the sequence of the Cas9-edited genomes of the $\operatorname{orc}^{Y 162 C}$ and orc $^{W T}$ strains are shown. The silent mutation removing the PAM site (gold), the Y162C mutation (maroon), and silent Ndel cut site (green) are noted along with the sequencing traces confirming the edited alleles. (C) The percent of balancer (CyO) to non-balancer adults are shown for the 462 crosses resulting in the indicated non-balancer progeny. Heterozygote males and females were mated, and their progeny were scored for the presence of the CyO balancer. The dashed gray line represents the expected ratio from this cross $(66 \%$ balancer: $33 \%$ nonbalancer). $\mathrm{n}=$ total number of flies assayed.

467 Figure 2: orc $4^{Y 162 C}$ animals have tissue-specific phenotypes (A) Images of homozygous orc4 ${ }^{W T}$ and orc $4^{Y 162 C}$ animals. Mutant animals have missing back bristles.

469 (top) and absent as well as disorganized wing-margin bristles (bottom). B. DAPI stained 470 images of stage 10 egg chambers from homozygous orc $4^{W T}$ and orc $4^{Y 162 C}$ animals.

471 Examples of orc $4^{\mathrm{Y} 162 \mathrm{C}}$ animals with disorganized follicle cells and reduced nurse cells are 472 shown. (C) Quantification of the number of nurse cells in orc $4^{W T}$, orc $4^{Y 162 C}$, and $m c m 6^{K 1214}$ 
473 ovaries. orc $4^{Y 162 C}$ females have significantly fewer nurse cells than both orc ${ }^{W T}(p<2.7$

$474 \times 10^{-17}, t$-test $)$ and $m c m 6^{K 1214}$ females $\left(p<1.0 \times 10^{-10}, t\right.$-test $)$. Fifty stage 10 chambers were

475 counted in orc4 ${ }^{W T}$ and $m c m 6^{K 1214}$ animals, 100 stage 10 chambers were counted in 476 orc4 $^{\mathrm{Y} 162 \mathrm{C}}$ animals.

477

478 Figure 3: orc4 ${ }^{\mathrm{Y162C}}$ females fail to replicate the chorion gene loci $(\mathrm{A}) \mathrm{EdU}$ staining of 479 stage $10 \mathrm{~B}$ egg chambers from orc ${ }^{W T}$, orc $4^{Y 162 C}$, and $m c m 6^{K 1214}$ females. EdU staining 480 (cyan) marks the amplified chorion genes and was acquired with identical settings for all 481 genotypes. DNA is stained with Hoechst (blue). Scale bar, $5 \mu \mathrm{m}$. (B) Quantification of 482 stage 10 chambers with EdU foci from orc $4^{W T}$, orc $4^{Y 162 C}$, and $m c m 6^{K 1214}$ females. 483 orc $4^{\mathrm{Y} 162 C}$ and $m c m 6^{K 1214}$ animals fail to amplify the chorion genes as compared to orc $4^{W T}$ 484 females $\left(p<1 \times 10^{-5}, t\right.$-test $)$. More than 50 stage 10 chambers were assayed for each 485 genotype.

487 Figure 4: Embryos inheriting maternal orc ${ }^{\mathrm{Y} 162 \mathrm{C}}$ are inviable. (A) Images of embryos 488 laid by mothers of the indicated genotype. WT (wild-type females); orc $4^{\mathrm{Y} 162 \mathrm{C}}$ (orc4 ${ }^{\mathrm{Y} 162 \mathrm{C}}$ 489 females); orc ${ }^{\mathrm{Y162C}}$ glc (heat shocked hsFLP122; FRTG13 ovo ${ }^{D 1 /}$ FRTG13 orc4 ${ }^{\text {Y162C }}$ 490 females with germline clones). Dorsal appendages are evident in embryos laid by wild491 type females and females heterozygous for orc $4^{Y 162 C}$ in the follicle cells (orc4 ${ }^{Y 162 C}$ glc), 492 but not from females homozygous for orc4 ${ }^{Y 162 C}$. (B) Average numbers of eggs laid from 493 ten females of the indicated genotypes over four days are shown. Error bars indicate the 494 standard deviation from three biological replicates. 


\section{References:}

Alvarez S., M. Diáz, J. Flach, S. Rodriguez-Acebes, A. J. López-Contreras, et al., 2015

Replication stress caused by low MCM expression limits fetal erythropoiesis and hematopoietic stem cell functionality. Nat. Commun. 6: 1-11.

https://doi.org/10.1038/ncomms9548

Balasov M., R. P. H. Huijbregts, and I. Chesnokov, 2009 Functional analysis of an Orc6 mutant in Drosophila. Proc. Natl. Acad. Sci. U. S. A. 106: 10672-10677. https://doi.org/10.1073/pnas.0902670106

Balasov M., K. Akhmetova, and I. Chesnokov, 2015 Drosophila model of Meier-Gorlin syndrome based on the mutation in a conserved C-Terminal domain of Orc6. Am.

J. Med. Genet. Part A 167: 2533-2540. https://doi.org/10.1002/ajmg.a.37214

Baldinger T., and M. Gossen, 2009 Binding of Drosophila ORC proteins to anaphase chromosomes requires cessation of mitotic cyclin-dependent kinase activity. Mol. Cell. Biol. 29: 140-149. https://doi.org/10.1128/MCB.00981-08

Bell S. P., R. Kobayashi, and B. Stillman, 1993 Yeast origin recognition complex functions in transcription silencing and DNA replication. Science 262: 1844-1849. https://doi.org/10.1126/science.8266072

Bicknell L. S., E. M. H. F. Bongers, A. Leitch, S. Brown, J. Schoots, et al., 2011a Mutations in the pre-replication complex cause Meier-Gorlin syndrome. Nat. Genet. 43: 356-360. https://doi.org/10.1038/ng.775

Bicknell L. S., S. Walker, A. Klingseisen, T. Stiff, A. Leitch, et al., 2011b Mutations in ORC1, encoding the largest subunit of the origin recognition complex, cause microcephalic primordial dwarfism resembling Meier-Gorlin syndrome. Nat. Genet. 
43: 350-356. https://doi.org/10.1038/ng.776

Bleichert F., M. Balasov, I. Chesnokov, E. Nogales, M. R. Botchan, et al., 2013 A Meier-

Gorlin syndrome mutation in a conserved C-terminal helix of Orc6 impedes origin recognition complex formation. Elife 2: 1-29. https://doi.org/10.7554/elife.00882

Burrage L. C., W.-L. Charng, M. K. Eldomery, J. R. Willer, E. E. Davis, et al., 2015 De

Novo GMNN Mutations Cause Autosomal-Dominant Primordial Dwarfism

Associated with Meier-Gorlin Syndrome. Am. J. Hum. Genet. 97: 904-913.

https://doi.org/10.1016/j.ajhg.2015.11.006

Calvi B. R., M. A. Lilly, and A. C. Spradling, 1998 Cell cycle control of chorion gene amplification. Genes Dev. 12: 734-744. https://doi.org/10.1101/gad.12.5.734

Chou T. B., E. Noll, and N. Perrimon, 1993 Autosomal P[ovoD1] dominant female-sterile insertions in Drosophila and their use in generating germ-line chimeras.

Development 119: 1359-1369.

Chou T. B., and N. Perrimon, 1996 The autosomal FLP-DFS technique for generating germline mosaics in Drosophila melanogaster. Genetics 144: 1673-1679.

Deshaies R. J., 1995 The self-destructive personality of a cell cycle in transition. Curr. Opin. Cell Biol. 7: 781-789.

Fenwick A. L., M. Kliszczak, F. Cooper, J. Murray, L. Sanchez-Pulido, et al., 2016 Mutations in CDC45, Encoding an Essential Component of the Pre-initiation Complex, Cause Meier-Gorlin Syndrome and Craniosynostosis. Am. J. Hum. Genet. 99: 125-138. https://doi.org/10.1016/j.ajhg.2016.05.019

Gorlin R. J., J. Cervenka, K. Moller, M. Horrobin, and C. J. J. Witkop, 1975 Malformation syndromes. A selected miscellany. Birth Defects Orig. Artic. Ser. 11: 39-50. 
Guernsey D. L., M. Matsuoka, H. Jiang, S. Evans, C. MacGillivray, et al., 2011

Mutations in origin recognition complex gene ORC4 cause Meier-Gorlin syndrome.

Nat. Genet. 43: 360-365. https://doi.org/10.1038/ng.777

Hamm D. C., E. D. Larson, M. Nevil, K. E. Marshall, R. Bondra, et al., 2017 A conserved maternal-specific repressive domain in Zelda revealed by Cas9-mediated mutagenesis in Drosophila melanogaster. 1-22.

https://doi.org/10.1371/journal.pgen.1007120

Hossain M., and B. Stillman, 2012 Meier-Gorlin syndrome mutations disrupt an Orc1 CDK inhibitory domain and cause centrosome reduplication. Genes Dev. 26: 17971810. https://doi.org/10.1101/gad.197178.112

Ilves I., T. Petojevic, J. J. Pesavento, and M. R. Botchan, 2010 Activation of the MCM27 helicase by association with Cdc45 and GINS proteins. Mol. Cell 37: 247-258. https://doi.org/10.1016/j.molcel.2009.12.030

Komitopoulou K., M. Gans, L. H. Margaritis, F. C. Kafatos, and M. Masson, 1983 Isolation and Characterization of Sex-Linked Female-Sterile Mutants in DROSOPHILA MELANOGASTER with Special Attention to Eggshell Mutants. Genetics 105: 897-920.

Landis G., R. Kelley, A. C. Spradling, and J. Tower, 1997 The k43 gene, required for chorion gene amplification and diploid cell chromosome replication, encodes the Drosophila homolog of yeast origin recognition complex subunit 2. Proc. Natl. Acad. Sci. U. S. A. 94: 3888-3892. https://doi.org/10.1073/pnas.94.8.3888

Landis G., and J. Tower, 1999 The Drosophila chiffon gene is required for chorion gene amplification, and is related to the yeast Dbf4 regulator of DNA replication and cell 
cycle. Development 126: 4281-4293.

Lee H. O., J. M. Davidson, and R. J. Duronio, 2009 Endoreplication: polyploidy with purpose. Genes Dev. 23: 2461-2477. https://doi.org/10.1101/gad.1829209

Maerz L. D., T. Casar Tena, J. Gerhards, C. Donow, P. A. Jeggo, et al., 2019 Analysis of cilia dysfunction phenotypes in zebrafish embryos depleted of Origin recognition complex factors. Eur. J. Hum. Genet. https://doi.org/10.1038/s41431-019-0338-0

MEIER Z., POSCHIAVO, and M. ROTHSCHILD, 1959 [Case of arthrogryposis multiplex congenita with mandibulofacial dysostosis (Franceschetti syndrome)]. Helv. Paediatr. Acta 14: 213-216.

Micklem G., A. Rowley, J. Harwood, K. Nasmyth, and J. F. Diffley, 1993 Yeast origin recognition complex is involved in DNA replication and transcriptional silencing. Nature 366: 87-89. https://doi.org/10.1038/366087a0

Moyer S. E., P. W. Lewis, and M. R. Botchan, 2006 Isolation of the Cdc45/Mcm27/GINS (CMG) complex, a candidate for the eukaryotic DNA replication fork helicase. Proc. Natl. Acad. Sci. U. S. A. 103: 10236-10241. https://doi.org/10.1073/pnas.0602400103

Munnik S. A. De, L. S. Bicknell, S. Aftimos, J. Y. Al-Aama, Y. Van Bever, et al., 2012 Meier-Gorlin syndrome genotype-phenotype studies: 35 individuals with prereplication complex gene mutations and 10 without molecular diagnosis. Eur. J. Hum. Genet. 20: 598-606. https://doi.org/10.1038/ejhg.2011.269 Okano-Uchida T., L. N. Kent, M. M. Ouseph, B. McCarty, J. J. Frank, et al., 2018 Endoreduplication of the mouse genome in the absence of ORC1. Genes Dev. 32: 978-990. https://doi.org/10.1101/gad.311910.118 
Orr-Weaver T. L., 1991 Drosophila chorion genes: cracking the eggshell's secrets.

Bioessays 13: 97-105. https://doi.org/10.1002/bies.950130302

Park S. Y., and M. Asano, 2008 The origin recognition complex is dispensable for endoreplication in Drosophila. Proc. Natl. Acad. Sci. U. S. A. 105: 12343-12348. https://doi.org/10.1073/pnas.0805189105

Park S. Y., and M. Asano, 2012 An orc1 allele with a mutated APC motif is female sterile with amplification defects. Cell Cycle 11: 2828-2832. https://doi.org/10.4161/cc.21168

Pflumm M. F., and M. R. Botchan, 2001 Orc mutants arrest in metaphase with abnormally condensed chromosomes. Development 128: 1697-1707.

Pinto S., D. G. Quintana, P. Smith, R. M. Mihalek, Z. H. Hou, et al., 1999 latheo encodes a subunit of the origin recognition complex and disrupts neuronal proliferation and adult olfactory memory when mutant. Neuron 23: 45-54.

Prasanth S. G., Z. Shen, K. V Prasanth, and B. Stillman, 2010 Human origin recognition complex is essential for HP1 binding to chromatin and heterochromatin organization. Proc. Natl. Acad. Sci. U. S. A. 107: 15093-15098. https://doi.org/10.1073/pnas.1009945107

Remus D., F. Beuron, G. Tolun, J. D. Griffith, E. P. Morris, et al., 2009 Concerted loading of Mcm2-7 double hexamers around DNA during DNA replication origin licensing. Cell 139: 719-730. https://doi.org/10.1016/j.cell.2009.10.015

Remus D., and J. F. X. Diffley, 2009 Eukaryotic DNA replication control: lock and load, then fire. Curr. Opin. Cell Biol. 21: 771-777. https://doi.org/10.1016/j.ceb.2009.08.002 
Sanchez J. C., E. X. Kwan, T. J. Pohl, H. M. Amemiya, M. K. Raghuraman, et al., 2017 Defective replication initiation results in locus specific chromosome breakage and a ribosomal RNA deficiency in yeast. PLoS Genet. 13.

https://doi.org/10.1371/journal.pgen.1007041

Sclafani R. A., and T. M. Holzen, 2007 Cell cycle regulation of DNA replication. Annu.

Rev. Genet. 41: 237-280. https://doi.org/10.1146/annurev.genet.41.110306.130308

Shu M., Y. Qin, and M. Jiang, 2008 RNA interference targeting ORC1 gene suppresses the proliferation of vascular smooth muscle cells in rats. Exp. Mol. Pathol. 84: 206212. https://doi.org/10.1016/j.yexmp.2008.03.001

Stiff T., M. Alagoz, D. Alcantara, E. Outwin, H. G. Brunner, et al., 2013 Deficiency in Origin Licensing Proteins Impairs Cilia Formation: Implications for the Aetiology of Meier-Gorlin Syndrome. PLoS Genet. 9.

https://doi.org/10.1371/journal.pgen.1003360

Stillman B., 2005 Origin recognition and the chromosome cycle. FEBS Lett. 579: 877884. https://doi.org/10.1016/j.febslet.2004.12.011

Tocilj A., K. F. On, Z. Yuan, and J. Sun, 2017 Structure of the active form of human origin recognition complex and its ATPase motor module. 1-23. https://doi.org/10.7554/eLife.20818

Vetro A., S. Savasta, A. Russo Raucci, C. Cerqua, G. Sartori, et al., 2017 MCM5: a new actor in the link between DNA replication and Meier-Gorlin syndrome. Eur. J. Hum. Genet. 25: 646-650. https://doi.org/10.1038/ejhg.2017.5

Whittaker A. J., I. Royzman, and T. L. Orr-Weaver, 2000 Drosophila double parked: a conserved, essential replication protein that colocalizes with the origin recognition 
complex and links DNA replication with mitosis and the down-regulation of S phase transcripts. Genes Dev. 14: 1765-1776.

Yao L., J. Chen, X. Wu, S. Jia, and A. Meng, 2017 Zebrafish cdc6 hypomorphic mutation causes Meier-Gorlin syndrome-like phenotype. Hum. Mol. Genet. 26: 4168-4180. https://doi.org/10.1093/hmg/ddx305 
bioRxiv preprint doi: https://doi.org/10.1101/711820; this version posted July 23, 2019. The copyright holder for this preprint (which was not certified by peer review) is the author/funder, who has granted bioRxiv a license to display the preprint in perpetuity. It is made available under aCC-BY-NC-ND 4.0 International license.

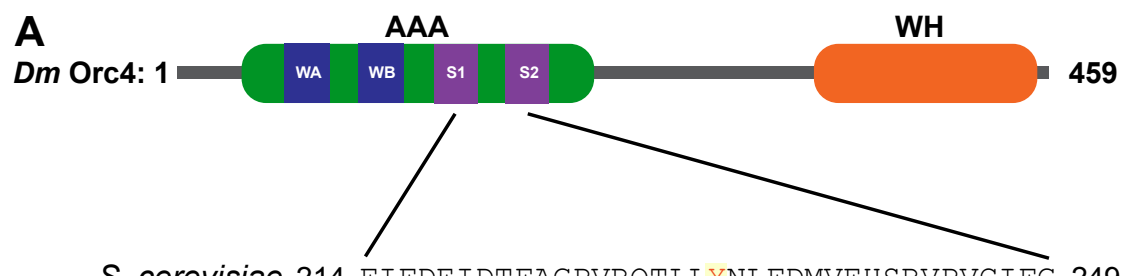

S. cerevisiae 214-FIFDEIDTFAGPVRQTLLYNLFDMVEHSRVPVCIFG -249

D. melanogaster 144-FILEEFDLFCAHHNQTLLYNLFDVSQSAQAP ICVLG -179

H. sapiens 156-F ILDEFDLFAHHKNQTLLYNLFDISQSAQTP IAVIG -191

M. musculus 153-FILDEFDIFAHQKNQTLLYNLFDISQSAQTPVAVIG -188

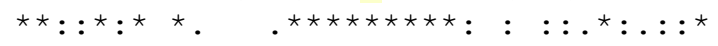

B

5' сCСtGCtCTACAACCTCTTCGACGTCTCCCAGTCAGCCCAGGCGCCCATCTGTG3' orc4 ${ }^{\text {Y162C }} 5$ 'СССtGCTTTGCAACCTCTTCGACGTCTCCCAGTCAGCCCAGGCGCCCATATGTG 3'
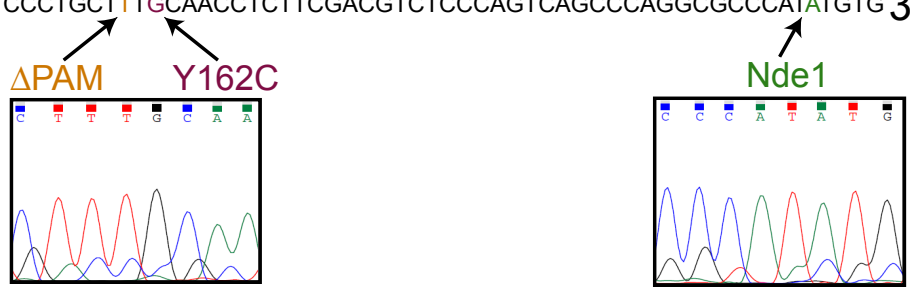

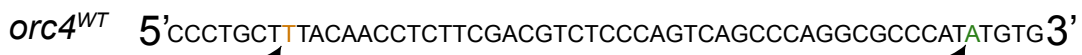
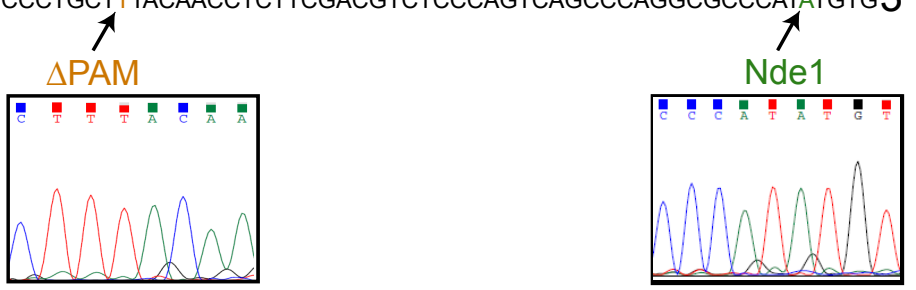

C

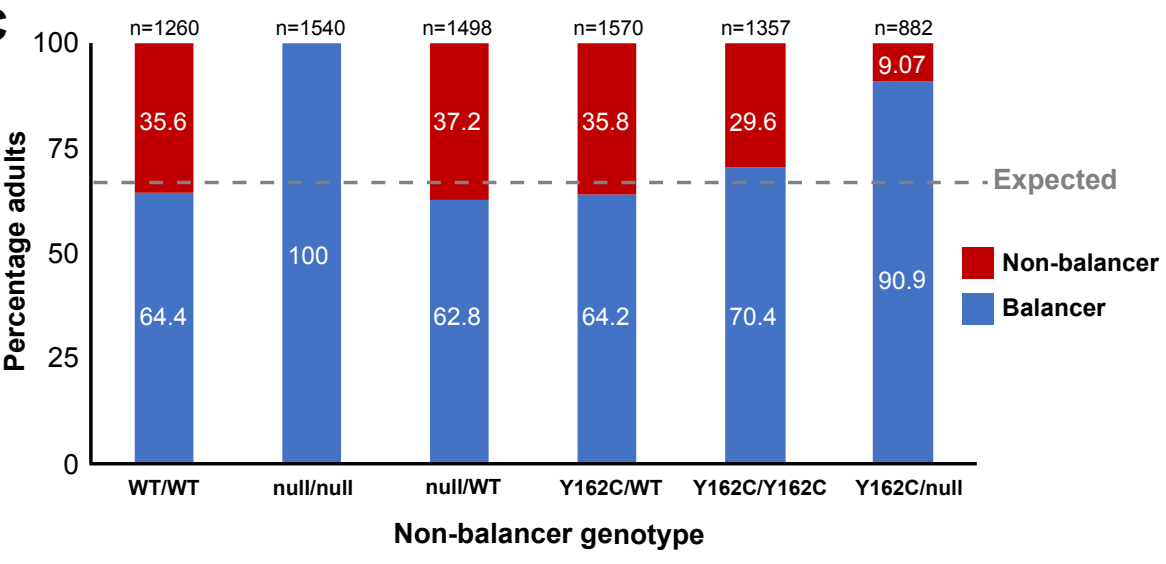


bioRxiv preprint doi: https://doi.org/10.1101/711820; this version posted July 23, 2019. The copyright holder for this preprint (which was not certified by peer review) is the author/funder, who has granted bioRxiv a license to display the preprint in perpetuity. It is made available under aCC-BY-NC-ND 4.0 International license.

A

$\operatorname{orc4} 4^{W T}$

orc $^{\text {Y162C }}$

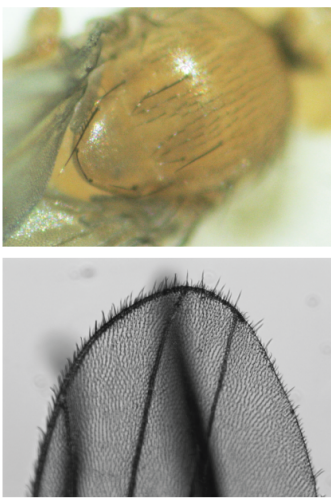

B

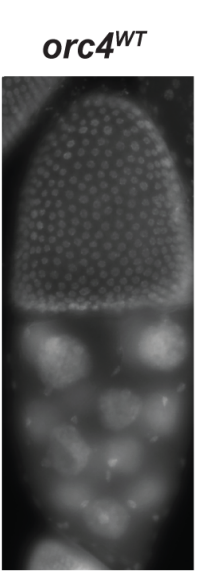

$\operatorname{orc}^{\mathrm{Y} 162 \mathrm{C}}$

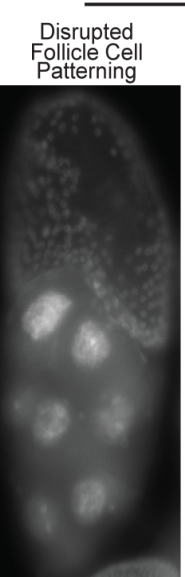

C

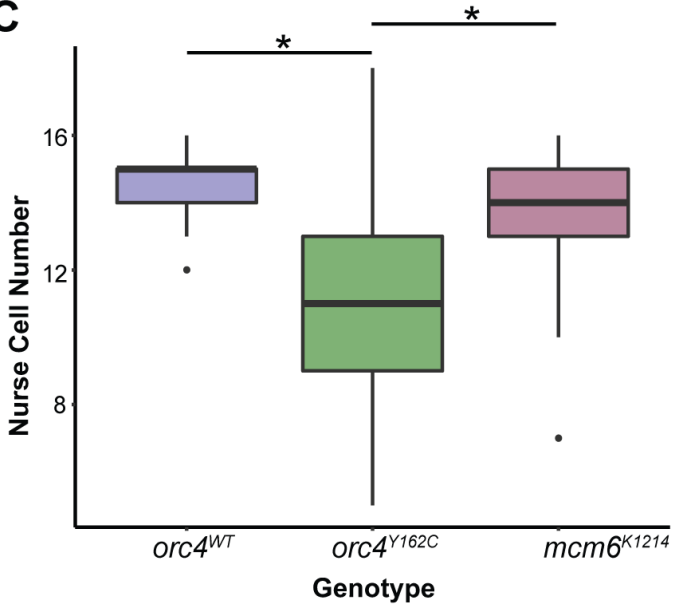


bioRxiv preprint doi: https://doi.org/10.1101/711820; this version posted July 23, 2019. The copyright holder for this preprint (which was not certified by peer review) is the author/funder, who has granted bioRxiv a license to display the preprint in perpetuity. It is made available under aCC-BY-NC-ND 4.0 International license.
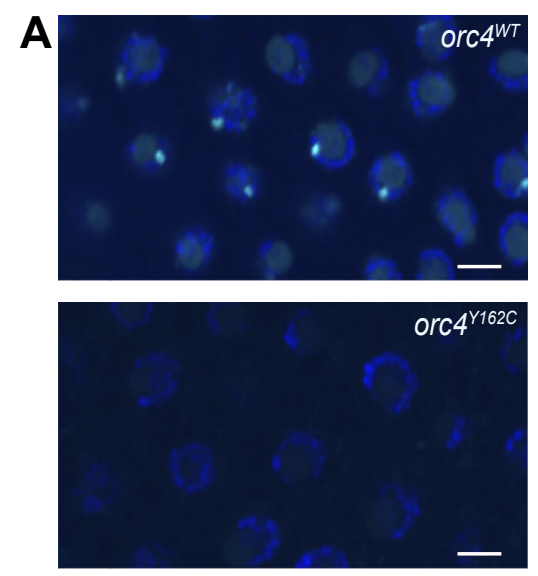

B
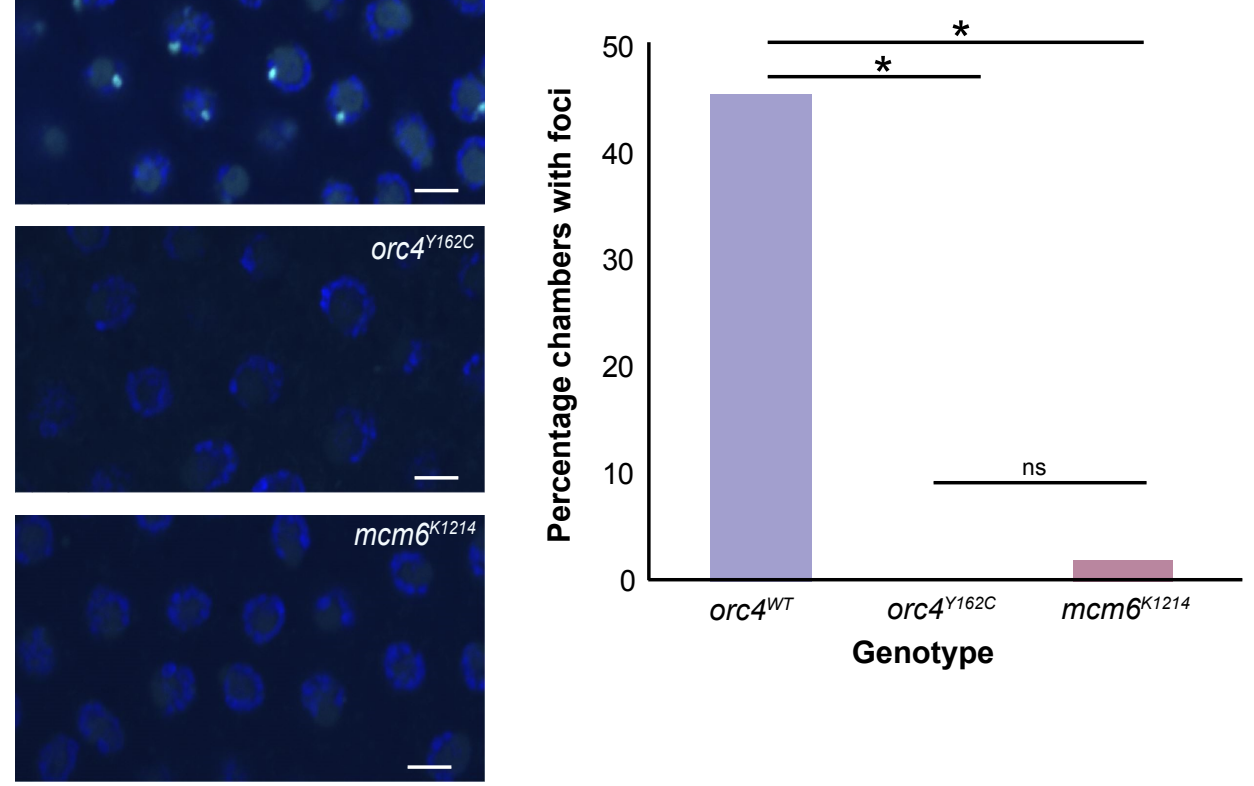
bioRxiv preprint doi: https://doi.org/10.1101/711820; this version posted July 23, 2019. The copyright holder for this preprint (which was not certified by peer review) is the author/funder, who has granted bioRxiv a license to display the preprint in perpetuity. It is made available under aCC-BY-NC-ND 4.0 International license.
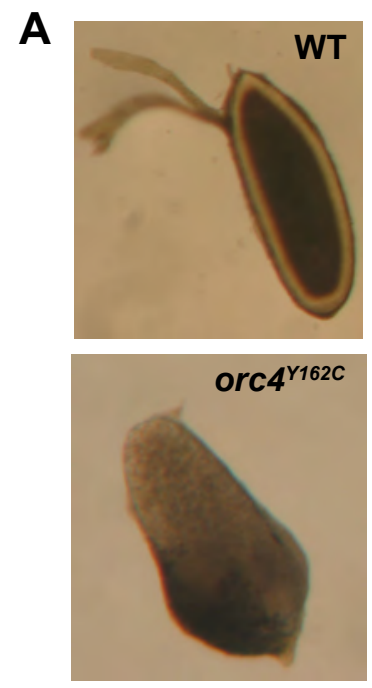
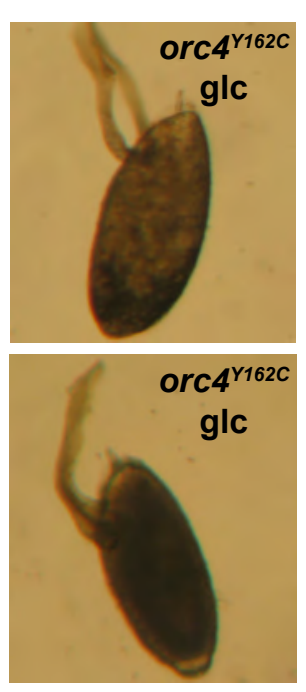

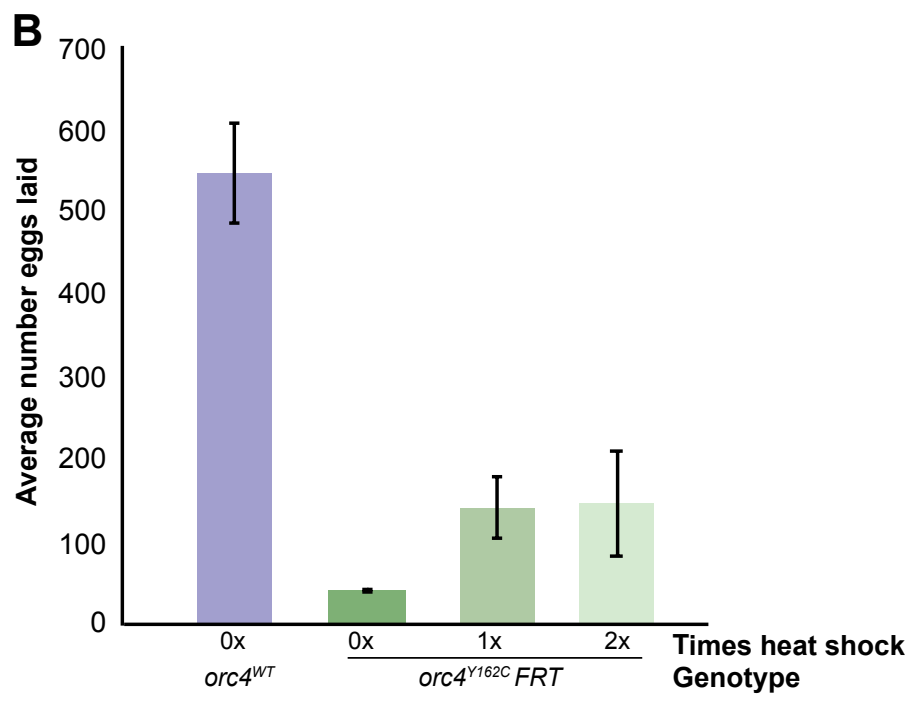

